\title{
Conformational Changes of Poly( $N$-isopropylacrylamide) Chains at Air/Water Interface: Effects of Temperature, Compression Rate and Packing Density
}

\author{
Guangming Liu, ${ }^{\dagger+\$}$ Shihe Yang*,† \\ ${ }^{\dagger}$ Department of Chemistry, The Hong Kong University of Science and Technology, Clear Water Bay, \\ Kowloon, Hong Kong, China \\ Guangzhao Zhang*,\$ \\ ${ }^{\dagger}$ Hefei National Laboratory for Physical Sciences at Microscale, Department of Chemical Physics, \\ University of Science and Technology of China, Hefei, Anhui, China
}

\section{Supporting Information}

\section{S-1. Packing density dependence of surface pressure of PS $_{126}-\mathrm{b}-\mathrm{PNIPAM}_{122}$ and PS $_{126}$-b-PNIPAM 243 at $\mathrm{T}=15^{\circ} \mathrm{C}, \mathrm{V}=5 \mathrm{~mm} / \mathrm{min}$.}

Figure S-1 shows packing density dependence of surface pressure of $\mathrm{PS}_{126}$-b-PNIPAM 122 and $\mathrm{PS}_{126}$-b-PNIPAM 243 at $\mathrm{T}=15^{\circ} \mathrm{C}, \mathrm{V}=5 \mathrm{~mm} / \mathrm{min}$. The sample with longer PNIPAM chains has a slightly larger surface pressure than that with shorter PNIPAM chains at the same packing density. This indicates that one isolated pancake unit of the longer PNIPAM chains occupies a much larger area than that of the shorter PNIPAM chains at the air/water interface. Similar results also were observed at other temperatures and compression/expansion rates (data not shown). 


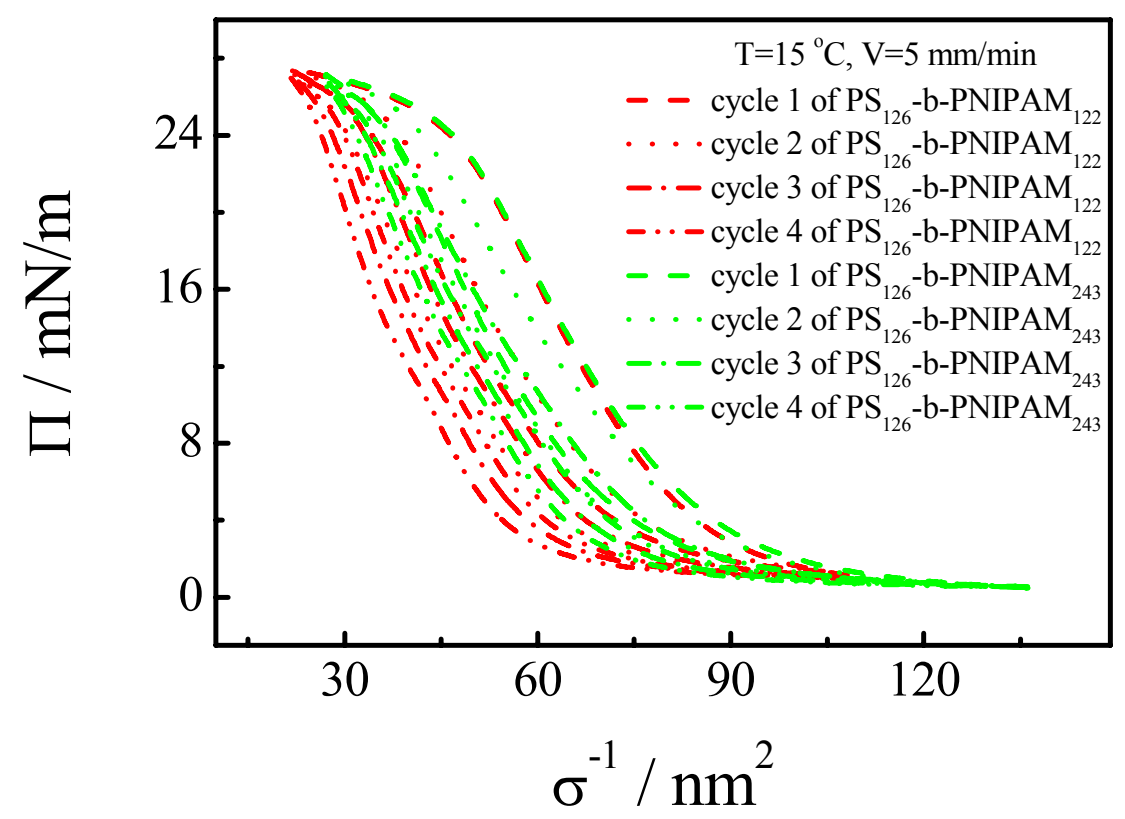

Figure S-1. $\Pi-\sigma^{-1}$ plots of $\mathrm{PS}_{126}-\mathrm{b}-\mathrm{PNIPAM}_{122}$ and $\mathrm{PS}_{126}-\mathrm{b}-\mathrm{PNIPAM}_{243}$ at $\mathrm{T}=15^{\circ} \mathrm{C}, \mathrm{V}=5$ $\mathrm{mm} / \mathrm{min}$.

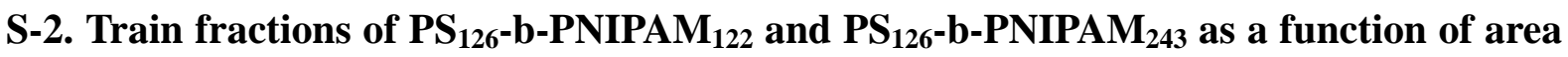 per molecule at different temperatures with $\mathrm{V}=5 \mathbf{m m} / \mathrm{min}$.}

From discussion in our paper, the PNIPAM chains adopt train conformation completely before and when the incompressibility reaches the maximum value. After the maximum, the polymer chains gradually switch to loop or tail conformation with increasing packing density accompanied by a gradual decrease of incompressibility (here the polymer chains have been assumed to adopt loops or tails completely when incompressibility drops to zero). Accordingly, the train fractions can be calculated at different temperatures from the plots of packing density dependence of incompressibility at different temperatures: train fraction $\%=$ $\left(\mathrm{C}^{-1} / \mathrm{C}^{-1} *\right) \times 100$, where $\mathrm{C}^{-1} *$ and $\mathrm{C}^{-1}$ are incompressibilities at the maximum and at a certain packing density after the maximum, respectively. Figure S-2 shows the train fractions of $\mathrm{PS}_{126}$-b-PNIPAM 122 and $\mathrm{PS}_{126}$-b-PNIPAM ${ }_{243}$ as a function of area per molecule at different temperatures with $\mathrm{V}=5 \mathrm{~mm} / \mathrm{min}$. Together with Figure $3(\mathrm{C}, \mathrm{D})$, it can be clearly seen that PNIPAM chains almost completely adopt the train conformation before the incompressibility 
reaches the maximum value $\left(\mathrm{PS}_{126}-\mathrm{b}-\mathrm{PNIPAM}_{122}: \sigma^{-1} \approx 60 \mathrm{~nm}^{2} ; \mathrm{PS}_{126}-\mathrm{b}-\mathrm{PNIPAM}_{243}: \sigma^{-1} \approx 65\right.$ $\mathrm{nm}^{2}$ ). In the low coverage region before the maximum, the train faction is assumed to be $100 \%$. Then the train fraction decreases with the decrease of area per molecule after the incompressibility maximum, indicating that the trains are gradually transformed to loops or tails. Besides, the train fraction has a slight increase with increasing temperature at a given area per molecule for the two samples, i.e., there exist more trains at the air/water interface at a higher temperature. This is consistent with the analysis from Figure 5, where surface pressure increases with temperature also because more trains exist at the air/water interface at a higher temperature.

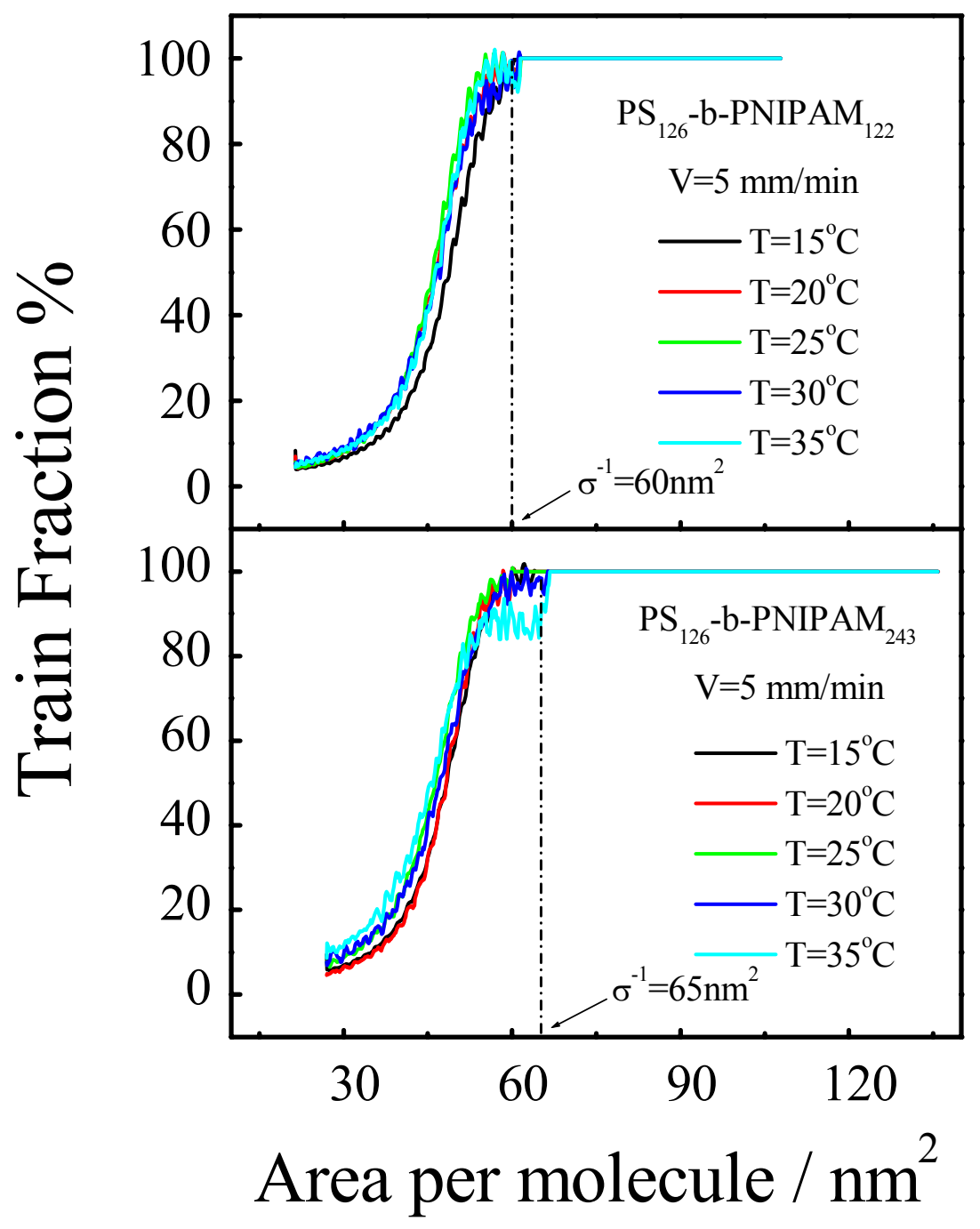

Figure S-2. Train fractions of $\mathrm{PS}_{126}$-b-PNIPAM 122 and $\mathrm{PS}_{126}$-b-PNIPAM 243 as a function of area per molecule at different temperatures with $\mathrm{V}=5 \mathrm{~mm} / \mathrm{min}$. 